Portland State University

PDXScholar

Mathematics and Statistics Faculty

Fariborz Maseeh Department of Mathematics

Publications and Presentations

and Statistics

2010

\title{
Symmetry and Stability of Homogeneous Flocks
}

J. J. P. Veerman

Portland State University, veerman@pdx.edu

Follow this and additional works at: https://pdxscholar.library.pdx.edu/mth_fac

Part of the Control Theory Commons, and the Non-linear Dynamics Commons Let us know how access to this document benefits you.

Citation Details

Veerman, J. J. P., "Symmetry and Stability of Homogeneous Flocks" (2010). Mathematics and Statistics Faculty Publications and Presentations. 131.

https://pdxscholar.library.pdx.edu/mth_fac/131

This Working Paper is brought to you for free and open access. It has been accepted for inclusion in Mathematics and Statistics Faculty Publications and Presentations by an authorized administrator of PDXScholar. Please contact us if we can make this document more accessible: pdxscholar@pdx.edu. 


\title{
SYMMETRY AND STABILITY OF HOMOGENEOUS FLOCKS A Position Paper
}

\author{
J. J. P. Veerman, \\ Fariborz, Maseeh Dept of Math. and Stat., Portland State University, Portland, OR 97201, USA \\ veerman@pdx.edu
}

Keywords: Coupled Oscillators, Network, Stability, Symmetry, Decentralized Control.

Abstract: The study of the movement of flocks, whether biological or technological, is motivated by the desire to understand the capability of coherent motion of a large number of agents that only receive very limited information. In a biological flock a large group of animals seek their course while moving in a more or less fixed formation. It seems reasonable that the immediate course is determined by leaders at the boundary of the flock. The others follow: what is their algorithm? The most popular technological application consists of cars on a one-lane road. The light turns green and the lead car accelerates. What is the efficient algorithm for the others to closely follow without accidents? In this position paper we present some general questions from a more fundamental point of view. We believe that the time is right to solve many of these questions: they are within our reach.

\section{INTRODUCTION}

We model agents and their interactions as a finite network of (coupled) oscillators in $\mathbb{R}^{d}$. We assume that agents observe relative positions and velocities only (no third and higher derivatives). The best studied example of such a flock is a class of well-known models for cars equipped with sensors and on automatic pilot that are moving in close formation on a one-lane road. However, we are interested in a wider class of flocks (especially those moving in $\mathbb{R}^{2}$ and $\mathbb{R}^{3}$ ). Our main concerns in this position paper fall in two categories: - What are the equations we should study?

- What does it mean for a (large) flock to be stable? These are the questions that motivate this position paper.In our view their will give rise to exciting new mathematics with ample implications for the sciences.

In response to the first concern we present physical considerations that impel us to consider translational symmetry of the equations of motion (in Section 2) and rotational symmetry and its breaking (in Section 3). These considerations are perhaps not fundamentally new: but the literature favors detailed studies that are of immediate technological importance. However, we argue that these equations can — and should - successfully be attacked.
The second concern is addressed in Section 4. We'll see that even in an asymptotically stable system, fluctuations may grow very large before they attenuate and die out. In one dimension they lead to collisions. In dimension greater than one, collisions are perhaps unlikely, but large fluctuations could lead to loss of cohesion of the flock (because the agents can't "see" each other anymore). Clearly there is a limit to the size of the fluctuations a flock can sustain. We will argue that it is useful to study this kind of stability in the time domain. We also argue that more general methods to investigate this kind of "transient (in)stability" should be researched.

We'll use a very simple set-up of a 1 dimensional flocks as our 'standard example'.

Definition 1.1 (Standard Example) Consider $N+1$ agents in $R$ labeled from 0 to $N$, each of whose coordinates are given by $z_{k}(t)$ as function of time. For $k \in\{1 \cdots, N-1\}$, let $f$ and $g$ be negative and $\rho$ and $r$ are in $(0,1)$.

$$
\begin{aligned}
\ddot{z}_{k}= & f\left\{z_{k}-(1-\rho) z_{k-1}-\rho z_{k+1}\right\} \\
& +g\left\{\dot{z}_{k}-(1-r) \dot{z}_{k-1}-r \dot{z}_{k+1}\right\}
\end{aligned}
$$

The boundary conditions are:

$$
\ddot{z}_{0}=0 \text { and } \ddot{z}_{N}=f\left\{z_{N}-z_{N-1}\right\}+g\left\{\dot{z}_{N}-\dot{z}_{N-1}\right\}
$$


The initial conditions are:

$\forall k: z_{k}(0)=0, \forall k>0: \dot{z}_{k}(0)=-0.1, \dot{z}_{0}(0)=0$.

It should be understood that the original coordinates of the agents given by $x_{k}$ where $x_{k}=z_{k}+h_{k}$; those are the coordinates we display in Figure 2. The $\left\{h_{k}\right\}$ are priori chosen constants and their differences determine the preferred relative positions of the agents.

\section{TRULY DECENTRALIZED LINEAR FLOCKS}

Ever since the inception ((Chandler et al., 1958), (Herman et al., 1959)) of the subject it has been a challenge to mathematically express the notion "decentralized" ((Chu, 1974)). Here is how we interpret it: In a decentralized flock the only information an agent receives are the position and velocity relative to it of some nearby agents. The agents do not receive information from an outside source. The only exceptions to this are some agents, called leaders, who may undergo an external forcing.

Proposition 2.1 (Galilean Invariance) In the absence of external forcing, the motion of a truly decentralized linear flock in $\mathbb{R}$ is described by ( $k$ and $i$ in $\{0 \cdots N\}$ ):

$$
\begin{aligned}
\ddot{x}_{k} & =f \sum_{i \in \mathcal{N}_{\rho}(k)} L_{\rho, k i}\left(x_{i}-h_{i}\right)+g \sum_{i \in \mathcal{N}_{r}(k)} L_{r, k i} \dot{x}_{i} \\
\text { or } \quad \ddot{z}_{k} & =f \sum_{i \in \mathcal{N}_{\rho}(k)} L_{\rho, k i} z_{i}+g \sum_{i \in \mathcal{N}_{r}(k)} L_{r, k i} \dot{z}_{i} .
\end{aligned}
$$

where $z_{k}=x_{k}-h_{k}$. The row-sums of the $N+1$ by $N+1$ "Laplacian" matrices $L_{\rho}$ and $L_{r}$ must be zero. The sums are over the set of agents $\mathcal{N}_{\varphi}$ whose relative velocity can be measured by $k$, resp. whose relative position can be measured by $K$ (in the case of $\mathcal{N}_{x}$ ). We furthermore have that there is a 2-parameter set of solutions given by (for any $Z$ and $V$ in $\mathbb{R})$ :

$$
\forall k \in\{0 \cdots, N\}: z_{k}(t)=V t+Z \quad .
$$

From now on we will always assume that $f$ and $g$ are chosen so that the system is asymptotically stable, that is: in the absence of external forcing, the system always converges to an orbit of the form $z_{k}(t)=V t+Z$. We call such a system stabilized. In the standard example, the stipulation that $f$ and $g$ be negative guarantees this. For general Laplacians, and especially in dimension bigger than 1, it not necessarily obvious how to achieve this and that question merits more attention.

In the following we denote $z \equiv\left(z_{0} \cdots, z_{N}\right)^{T}$ in $\mathbb{C}^{N+1}$. Similarly $a$ is in $\mathbb{C}^{N+1}$.
Lemma 2.2 Suppose in the system of Proposition 2.1 agent " 0 " is the only leader and its orbit is given by $z_{0}(t)=e^{i \omega t}$. Then as $t \rightarrow \infty$ the orbit of the system converges to

$$
\begin{aligned}
& \qquad(t)=a e^{i \omega t}, \\
& \text { where Galilean Invariance implies that } \\
& \begin{array}{l}
a=\left(a_{0} \cdots, a_{N}\right) \in \mathbb{C}^{n} \text { satisfies: } \\
-\omega^{2} a=\left(f L_{\rho}+i \omega g L_{r}\right) a
\end{array}
\end{aligned}
$$

And in particular $\lim _{\omega \rightarrow 0} a_{k}(\omega)=1$ for all $k \in$ $\{0 \cdots, N\}$.

This Lemma is immediately relevant to the present discussion because it indicates that certain lowfrequency perturbations in the orbit of the leader cannot possibly die out: it is impossible to design a decentralized system where all perturbations die out. The situation is in fact probably worse: as the size of the system grows, it seems that, say, $\max _{\omega \in \mathbb{R}^{+}}\left|a_{N}(\omega)\right|$ must also grow, probably with some power $1 / d$ of the number $N$ of agents, where perhaps $d$ is related to a notion of the dimensionality of the "communication graph". This question has received some attention ((Pant et al., 2002)), but not nearly enough.

The trouble doesn't even end there. When we study flocks - whether biological or technological - it is natural to assume that each agent performs the same computation to determine its acceleration. When you imagine a large flock then for all agents 'sort of' in the middle of that flock that assumption is reasonable: all agents are hardwired equal. We'll call this homogeneity. It is hard to give a formal definition of this concept since it cannot be completely true for a finite flock moving in $\mathbb{R}^{d}$ : the agents at the physical boundary of the flock necessarily have less neighbors. Since perturbations cannot all die out - as we just showed, the boundary conditions may well influence the dynamics of the flock. We can see two possibilities: either boundary conditions (or some natural subset thereof) don't matter, or else there is a smart and natural way of uniquely specifying them. To the best of our knowledge this important question has hardly if ever been addressed (except (Sullivan, 2010), unpublished)!

\section{DECENTRALIZED FLOCKS IN A MEDIUM, MASS}

We do not live in a vacuum! Whatever our method of locomotion is, in a medium we can perceive our speed with respect to the medium, by the effort we expend to maintain the speed to overcome friction. (Though without a compass we cannot measure the 
direction of that speed.) The consequence of perceiving speed is that there is a preferred direction for the agents, namely: forward (in the direction of the velocity). Thus flocks in dimension 2 can orient themselves so that their desired configuration is aligned along the velocity of the flock.

To formulate those equations in $\mathbb{R}^{2}$ let's go back to the system given in Proposition 2.1 in the coordinates of the agents given by $x_{k}$ where $x_{k}=z_{k}+h_{k}$ (the first of the two equations). We now interpret $x_{k}$ and $h_{k}$ as vectors in $\mathbb{R}^{2}$. These equations are not truly decentralized anymore! An agent unsure where North is can only measure $\left|h_{k}-h_{k-1}\right|$ (ie: relative distances). However flying in a medium, the agent can perceive their direction of flight and orient itself according to it (as long as their speed is non-zero.) Thus the rotational symmetry of the truly decentralized equations in dimension 2 can be broken. We argue that this is how geese manage to fly in formation. Here are the equations:

Proposition 3.1 ((Veerman et al., 2005)) Let $\theta_{k}$ be angle that $\dot{x}_{k}$ makes with the positive $x$-axis. Denote by $R_{\theta}$ the rotation by $\theta$. The equation of motion for the orientable flock is:

$$
\ddot{x}_{k}=f \sum_{i \in \mathcal{N}_{\rho}(k)} L_{\rho, k i}\left(x_{i}-R_{\theta_{k}} h_{i}\right)+g \sum_{i \in \mathcal{N}_{r}(k)} L_{r, k i} \dot{x}_{i},
$$

as long as all $\dot{x}_{i}$ are non-zero. (The equation has a singularity at $\dot{x}_{i}=0$.)

In Figure 1 taken from (Veerman et al., 2005) we show a numerical simulation using the Equations of Proposition 3.1. The figure shows that in principle it is possible to re-orient a (mathematical) flock as it turns. (Remark: The way we did it in that paper is not exactly how we propose to study that question here. But it does provide a proof of concept.) (See also (Williams et al., 2005)) The important question is: how can we understand the re-alignment of a flock in 2 (or 3 ) dimensions that changes its direction when certain leaders at its boundary initiate a course change. We invite the reader to study these equations! The non-linearity makes these equations a real challenge to study (a few results are given in (Veerman et al., 2005)).

This leaves two other questions. It is possible to introduce a desired speed $V$ in the above equations. Furthermore in a realistic flock it is likely that the masses $m_{k}$ of the agents have a distribution with average 1 (as opposed to being exactly 1 ). The equations (in the absence of any external forces) then become:

$$
\begin{aligned}
m_{k} \ddot{x}_{k} & =f \sum_{i \in \mathcal{N}_{\varphi}(k)} L_{\rho, k i}\left(x_{i}-R_{\theta_{k}} h_{i}\right)+ \\
& g \sum_{i \in \mathcal{N}_{k}(k)} L_{r, k i} \dot{x}_{i}+\alpha\left(1-\frac{V}{\left|\dot{x}_{k}\right|}\right) \dot{x}_{k} .
\end{aligned}
$$

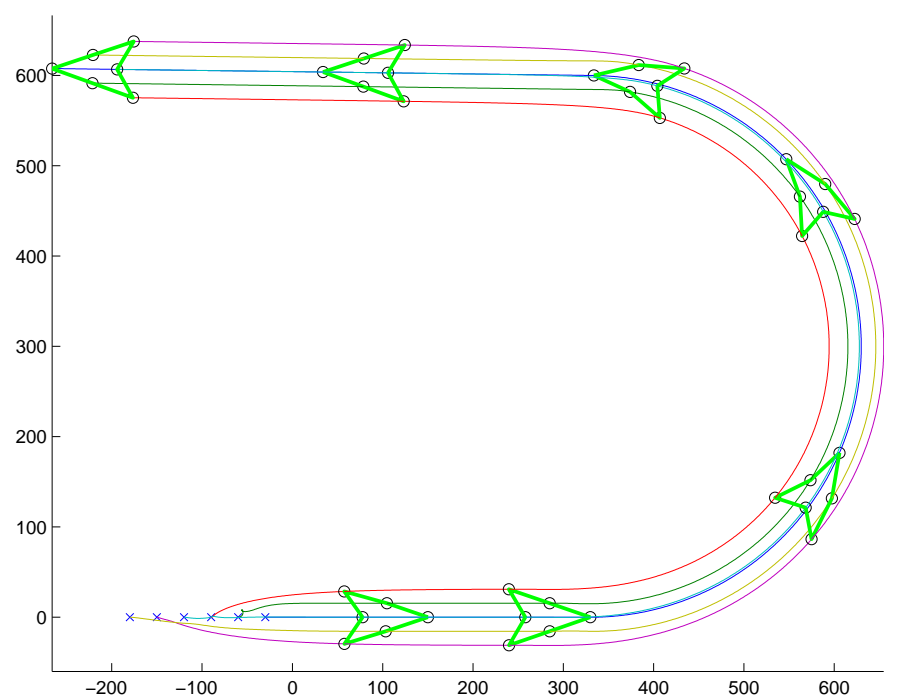

Figure 1: (color online) This figure is a simulation of a flock turning around using Definition 3.1. Note that the orientation of the flock's configuration changes. Each 'Christmas tree' shaped hexagon is a snapshot of the position of the flock, the lines that form the figure only facilitate visual inspection, they have no physical or mathematical content.

Here $\alpha<0$. We expect that both the presence of desired speed (a cruise speed) and the variation in the masses act to stabilize the equations to some extent. In a realistic setting we probably want flocks to be able to travel at different speeds: in different environments (say, cities or freeways) the desired cruise speeds should be different. Furthermore even when we agree on a single desired cruise speed, it appears unrealistic that individual agents in dense traffic can be programmed to determine their absolute speed accurately enough so as to avoid collisions with their immediate neighbors. Nonetheless we offer Equation 1 as a possible starting point for a more general mathematical study of flocking in 2 dimensions than the equation in Proposition 3.1.

\section{SYMMETRY AND STABILITY}

Now we come to what is certainly one of the most intriguing and important questions concerning flocks: What happens if a member at the "boundary" of the flock suddenly its velocity for whatever reason (a predator is observed or a traffic light changes etc)? How is the capability of following the new - supposedly more advantageous - affected by the size of the flock? The (in)ability to effectively follow the new course should limit the maximum allowable size of the flock. What is that size, and how can we imple- 
ment that in a 'technological' flock? Recall that we assume that a flock is asymptotically stable, so that no matter what the course change is, the other agents will ultimately follow the leader. The catch is that if, prior to stabilizing, fluctuations become too large: the flock is still not viable. Sometimes this is informally referred to as 'transient stability'. In the literature the concept of 'string stability' has played an important role ((Swaroop and Hedrick, 1996a), (Swaroop and Hedrick, 1996b)).

Let's look at the Standard Example (Definition 1.1). That system is given in such a way that $\lim _{t \rightarrow \infty} z(t)=0$ and $\lim _{t \rightarrow \infty} \dot{z}(t)=0$. This is important in what follows. Consider the quantities $\max _{k} \max _{\omega \geq 0}\left|a_{k}(\omega)\right|$ and of $\max _{k} \max _{t \geq 0}\left|z_{k}(t)\right|$. It turns out that the maxima are achieved for $k=N$, which simplifies the notation.

Definition 4.1 We call the system 'harmonically unstable' if

$$
\frac{1}{N} \ln \max _{\omega \geq 0}\left|a_{N}(\omega)\right|>0 \quad,
$$

and 'impulse unstable' if

$$
\frac{1}{N} \ln \max _{t \geq 0}\left|z_{N}(t)\right|>0 .
$$

The system is 'flock unstable' if either holds, 'flock stable' otherwise.

Theorem 4.2 ((Veerman et al., 2009), (Veerman, 2010), (Veerman and Tangerman, 2010), (Tangerman et al., 2010)) The system given in Definition 1.1 with $r=\rho$ is harmonically and impulse stable $\rho=1 / 2$ and harmonically and impulse unstable for $\rho \in(0,1) \backslash\{1 / 2\}$.

There are several problems with this. To set up the definition of flock stability more generally, one would have to rely on 'homogeneity' which is hard to define (see last paragraph of Section 2). The second problem is that the Theorem is so hard to prove that it seems unlikely that substantial generalizations can be made - at least not using the methods of those papers.

The effect of changing $\rho$ are truly dramatic as the accompanying figures show. (This was also observed in (Barooah and Hespanha, 2005).) In Figure 2 (taken from (Tangerman et al., 2010)) we show a simulation of the system of Definition 1.1 with $r=\rho$ and with 100 agents, and only slightly vary $\rho: 0.45,0.50$, and 0.55 . For $\rho<1 / 2$ wild fluctuations of relatively high frequency occur wiping out every semblance of cohesion in short order. For $\rho>1 / 2$ a very large fluctuation occurs over a very large time-scale (a factor $10^{3}$ longer than we can exhibit here). As indicated before, both systems ultimately stabilize: in practice this is moot, because the transient fluctuations would have destroyed cohesion of any actual physical flock already. In Figure 3 we show (the absolute value of) the frequency response functions in the three cases: $\rho=0.45, \rho=1 / 2$, and rho $=0.55$. Note the difference in scales! When $\rho=1 / 2$ the spectrum the frequency response functions have a very peculiar form that enables us to deduce the actual dynamics of the individuals. They undergo a damped stop-and-go motion (analyzed in (Veerman et al., 2009) and reminiscent of post-holiday traffic into urban areas).

These results reveal yet another curious fact. In finite-dimensional (linear) systems we are used to the intuition that large fluctuations occur when the eigenvalues associated with the first order ODE (the poles of $a_{N}(\omega)$ ) get close to the imaginary axis. Here we vary the dimension $N$ of the system and now this intuition turns out to be completely false! In this case we can calculate the eigenvalues of the system (see (DaFonseca and Veerman, 2009) and (Tangerman et al., 2010)). The results are displayed in Figure 4. When $\rho>1 / 2$ there is an eigenvalue (indicated in red) extremely close (exponentially in $N$ ) to 0 ; that eigenvalue dominates the behavior of the system. When $\rho=1 / 2$ there are a couple of eigenvalues roughly at distance $N^{-2}$ of the imaginary axis; these few also dominate the dynamics. However when $\rho<1 / 2$ all eigenvalues are bounded away from the imaginary axis, uniformly in $N$ ! Now all eigenvalues contribute to the dynamics and this gives surprising results: very large fluctuations. From the point of view of studying the eigenvalues, this is completely counter-intuitive.

In view of these difficulties we close by encouraging (again) the search for a more general point of view. Let's go back to the more general system given in Proposition 2.1. The physical "work" $W_{k}$ done by the forces acting upon agent $k$ between time 0 and time $t$ is given by $\int_{0}^{t} \ddot{z}_{k} \dot{z}_{k} d t$ which equals $\frac{1}{2}\left(\dot{z}_{k}^{2}(t)-\dot{z}_{k}^{2}(0)\right)$. Employing $(\cdot, \cdot)$, the usual Hermitian inner product on $\mathbb{C}^{N+1}$, the sum of the $W_{k}$ 's can be written as $\frac{1}{2}((\dot{z}, \dot{z})(t)-(\dot{z}, \dot{z})(0))$. After some algebra one obtains for arbitrary Laplacians $L_{\rho}$ and $L_{r}$ :

Proposition 4.3 The equations given in Proposition 2.1 imply:

$$
\begin{aligned}
& \frac{1}{2}\left[(\dot{z}, \dot{z})(t)-f\left(L_{\rho}^{S} z, z\right)(t)\right]= \\
& \quad \frac{1}{2}\left[(\dot{z}, \dot{z})(0)-f\left(L_{\rho}^{S} z, z\right)(0)\right] \\
& \quad+g \int_{0}^{t}\left(L_{r}^{S} \dot{z}, \dot{z}\right) d t+f \int_{0}^{t}\left(L_{\rho}^{A} z, \dot{z}\right) d t .
\end{aligned}
$$

Here for a matrix $L$ we have written $L=L^{S}+L^{A}$, the latter two denoting the symmetric and the antisymmetric parts.

Specialize to the Standard Example. One easily sees that $(\dot{z}, \dot{z})(0)=N$ while $\left(L_{\rho}^{S} z, z\right)(0)=0$. Perhaps 

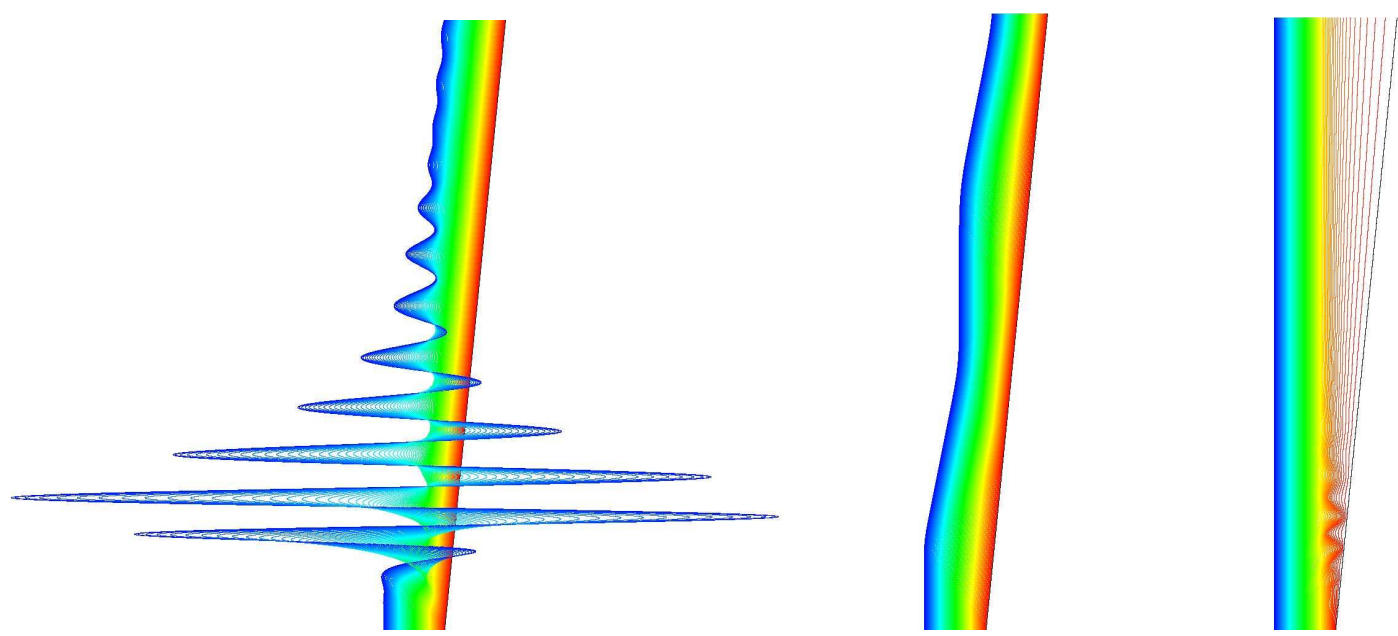

Figure 2: (color online) Numerical solution of the equation of motion of Definition 1.1 with $r=\rho, f=-1, g=-2, N=100$. Time $(t)$ is vertical and position $(x)$ is horizontal, each Figure being approximately a 1000 by 1000 square. Each agent initially has unit distance to its neighbors, so that the flock initially is 100 units wide (horizontally). At $t=0$ the rightmost agent, the leader, starts moving with constant velocity 0.1 to the right. Simulations for $\rho=0.45$ (left), $\rho=0.50$ (middle), and $\rho=0.55$ (right).
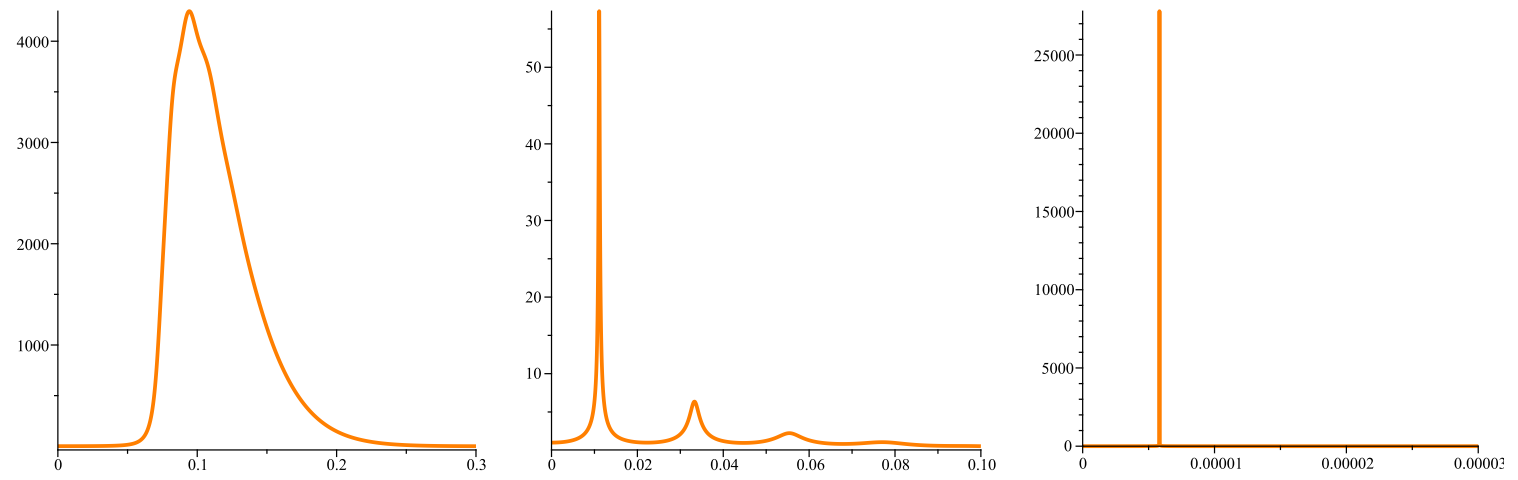

Figure 3: (color online) The frequency response function for the system of Figure 2. The absolute value of $a_{N}(\omega)$ for $\rho=0.45$ (left), $\rho=0.50$ (middle), and $\rho=0.55$ (right). Observe the different scales in these Figures!
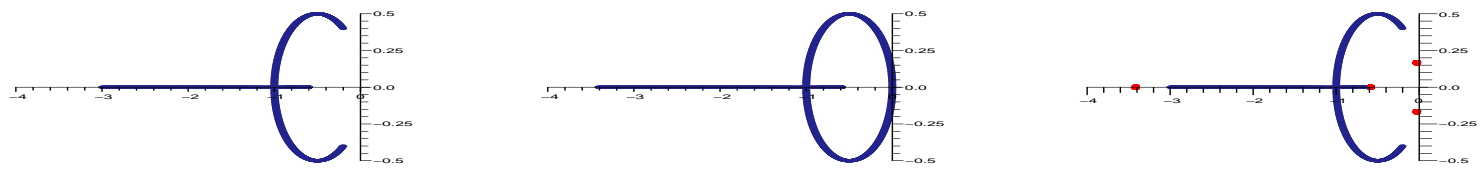

Figure 4: (color online) Schematic representation in the complex plane of the eigenvalues associated to the first order ODE of the system given in Figure 2. For $\rho<0.5$ the real part of the eigenvalues are less than a negative constant (right). When $\rho$ approaches 0.5 , the eigenvalues approximate the imaginary axis and the origin (middle). When $\rho>0.5$ the process reverses, except that two pairs of eigenvalues (in red) is spun off. One of these is exponentially (in $N$ ) close to zero (left). 
somewhat surprisingly, the matrices $L_{\rho}^{S}$ and $L_{r}^{S}$ have one negative eigenvalue (due to the special form of the equation of motion of the leader). This eigenvalue appears to have small modulus. Similarly, the matrix $L_{\rho}^{A}$ has non-zero entries only near the 'boundary-terms'. Thus the left-hand side tries to be semi-definite positive while the last two terms of right-hand side is "try" to be negative, pushing the right-hand side to zero, but boundary effects slow that down. A more careful analysis would be extremely interesting. The new aspect is that Proposition 4.3 is valid is completely independent of the topology of the network. We expect that similar equations can be written for flocks in dimension 2 or 3 .

\section{CONCLUSION}

The first point we argue in this position paper is that considerations of a fundamental nature (symmetry, friction) can help lead us to formulate the simplest possible equations governing the motion of decentralized flocks. In one dimension these are linear (Proposition 2.1), but in higher dimension that is not the case (Section 3). We contend that these equations (especially the ones in dimension 2 or 3 ) have been insufficiently studied in favor of ad hoc models. For example it is not well-known enough that perturbations cannot all die out as they travel through the flock (Lemma 2.2. Neither do we know of any detailed studies of flocks in $\mathbb{R}^{2}$ or $\mathbb{R}^{3}$ that change direction and orientation s suggested in Section 3.

The second point we bring up is that of flockstability. In Definition 4.1 we propose that the stability of a flock is determined by how certain response functions grow with the size of the (homogeneous) flock. A fundamental result, Theorem 4.2, states flock stability is intimately related to internal symmetry of the flock. This result together with Figure 4 shows that it is completely misleading to look at the location of the eigenvalues for these linear systems: one must look at the time domain as well (the impulse response function of Definition 4.1). This is not widely acknowledged in the literature. Since time-domain results as those of Theorem 4.2) are so hard to obtain, the need for new methods - preferably based on general principles - is great. In Proposition 4.3 we propose to gain some insight in a general setting by evaluating the physical "work" done by the system.

\section{ACKNOWLEDGEMENTS}

I am grateful to Borko Stosic for numerous discussions.

\section{REFERENCES}

Barooah, P. and Hespanha, J. P. (2005). Error amplification and disturbance propagation in vehicle strings with decentralized linear control. In IEEE conference on decision and control, volume 5, pages 4964-4969.

Chandler, R. E., Herman, R., and Montroll, E. W. (1958). Traffic dynamics: Studies in car following. Op. Res., 6:165-184

Chu, K.-C. (1974). Decentralized control of high-speed vehicle strings. Transportation Science, 8(4):361-384.

DaFonseca, C. M. and Veerman, J. J. P. (2009). On the spectra of certain directed paths. Appl. Math. Lett., 9:1351-1355.

Herman, R., Montroll, E. W., Potts, R. B., and Rothery, R. W. (1959). Traffic dynamics: Analysis and stability in car following. Op. Res., 7:86-106.

Pant, A., Seiler, P., and Hedrick, K. (2002). Mesh stability of look-ahead systems. IEEE Trans Aut. Contr., 47(2):403-408.

Sullivan, W. (2010). Boundary Conditions and a One Lane Linear model of Traffic Flow. Portland State University, Master's Thesis.

Swaroop, D. and Hedrick, K. (1996a). String stability of interconnected systems. IEEE Trans. Aut. Contr., 41(3):349-357.

Swaroop, D. and Hedrick, K. (1996b). String stability of interconnected systems. IEEE Trans. Aut. Contr., 41(3):349-357.

Tangerman, F. M., Veerman, J. J. P., and Stosic, B. D. (2010). Asymmetric decentralized flocks. Submitted.

Veerman, J. J. P. (2010). Stability of large flocks: an example. arXiv:1002.0768.

Veerman, J. J. P., Caughman, J. S., Lafferriere, G., and Williams, A. (2005). Flocks and formations. J. Stat. Phys., 121(5-6):901-936.

Veerman, J. J. P., Stosic, B. D., and Tangerman, F. M. (2009). Automated traffic and the finite size resonance. J. Stat. Phys., 137(1):189-203.

Veerman, J. J. P. and Tangerman, F. M. (2010). Impulse stability of large flocks: an example. arXiv:1002.0782.

Williams, A., Lafferriere, G., and Veerman, J. (2005). Stable motions of vehicle formations. In IEEE 44th Conf. on Decision and Contr., pages 72-77. 